\title{
Morfología vegetal como herramienta para la identificación de adulterantes en los frutos comercializados como "anís verde", Pimpinella anisum L. (Apiaceae)
}

Morfologia vegetal como ferramenta para a identificação de adulterantes em frutos comercializados como "anis verde", Pimpinella anisum L. (Apiaceae)

Plant morphology as a tool for the identification of adulterants in the fruit marketed as "anise", Pimpinella anisum L. (Apiaceae)

Recebido em: 05/04/2018 Aceito em: 13/06/2018 Facultad de Ciencias Químicas, Universidad Nacional de Asunción, Campus Universitario, San Lorenzo. Casilla de correos 11001-3291.

Asunción.Paraguay.E-mail:yenny07@gmail.com

\section{ABSTRACT}

Plant morphology as a tool for the identification of adulterants in the fruit marketed as "anise", Pimpinella anisum L. (Apiaceae). Paraguay is characterized by a population that consumes largely medicinal plants for the treatment of various conditions. However, in our country there is not well-established legislation regarding the control of these products and, within this context, the question arises: the population knows what is consuming? Such situation creates a fertile ground for adulteration and substitution of all kinds. This work was developed within the framework of the project: "Identification of adulterants and foreign matter in medicinal fruits, marketed in main herbalists of Asuncion and Great Asuncion, as a contribution to the quality control of herbal drugs", funded by the Rectorado of the National University of Asuncion, and aimed to determine the presence of adulterants in the fruits marketed as "anise" Pimpinella anisum L. in main herbalists of Asuncion and Great Asuncion, using plant morphology as a tool. For this sample, the fruits of "anise" of herbalists were acquired and then analyzed in the laboratory. Two adulterants species of "anise" were identified: seeds of "sesame", Sesamum indicum and fruits of "kuratú" Coriandrum sativum; all samples showed in his one of these adulterants or even both composition, however, all sold at different prices, some even three times the value than others. Thus, morphological analysis can provide useful data when performing quality control of herbal drugs, to provide population security in what is consuming.

Keywords: "anise"; Pimpinella anisum; adulterants; quality control; medicinal fruits

\section{RESUMEN}

El Paraguay es un país caracterizado por una población que consume en gran medida plantas medicinales para el tratamiento de diversas afecciones. Sin embargo, en nuestro país no hay un legislación bien definida respecto al control de este tipo de productos y, dentro de este contexto surge la interrogante: sabe la población lo que está consumiendo?. Esto, crea un terreno fértil para la adulteración y sustitución de todo tipo. El presente trabajo se 
desarrolló dentro del marco del Proyecto: "Identificación de adulterantes y materias extrañas en frutos medicinales, comercializados en principales herboristerías de Asunción y Gran Asunción, como aporte para el control de calidad de drogas vegetales", financiado por el Rectorado de la Universidad Nacional de Asunción, y tuvo como objetivo, determinar la presencia de adulterantes en los frutos comercializados como "anís verde", Pimpinella anisum L., en las principales herboristerías de Asunción y Gran Asunción, empleando la morfología vegetal como herramienta. Para ello se adquirieron las muestras de los frutos de "anís verde" de las herboristerías, y luego fueron analizadas en el laboratorio. Como resultado se identificaron en total dos especies adulterantes del "anís verde": semillas de sésamo, Sesamun indicum y frutos de "kuratú", Coriandrum sativum; todas las muestras presentaron en su composición alguno de estos adulterantes o incluso ambos, sin embargo, todas se comercializan a diferentes precios, algunas incluso al triple del valor que otras. De esta manera, el análisis morfológico, permite aportar datos útiles a la hora de realizar el control de calidad de drogas vegetales, de manera a brindar a la población, seguridad en lo que está consumiendo. Palabras clave: "anís verde"; Pimpinella anisum; adulterantes; control de calidad; frutos medicinales.

\section{RESUMO}

O Paraguai é um país caracterizado por uma população que consome, em grande parte, plantas medicinais para o tratamento de várias condições. No entanto, em nosso país não existe uma legislação bem definida sobre o controle desse tipo de produto e, nesse contexto, surge a pergunta: a população sabe o que está consumindo? Isso cria um terreno fértil para adulteração e substituição de todos os tipos. Este trabalho foi realizado no âmbito do projeto: "Identificação de adulterantes e matérias estranhas em frutas medicinais, que são vendidos em grandes herboristerías de Assunção e Grande Assunção, como uma contribuição para o controle de drogas à base de plantas de qualidade", financiado pela Reitoria da Universidade Nacional de Assunção, e teve como objetivo determinar a presença de adulterantes nos frutos comercializados como "anis" Pimpinella anisum L., nos principais herbanários de Assunção e Grande Assunção, usando a morfologia da planta como uma ferramenta. Para isso, amostras de frutos de "anis verde" foram adquiridas, e analisadas em laboratório. Como resultado, foram identificadas duas espécies adulterantes de "anis verde": sementes de gergelim, Sesamun indicum, e frutos de "kuratú", Coriandrum sativum. Todas as amostras analisadas continham ao menos um desses adulterantes. Ainda, uma grande variação de preço foi observada no momento da aquisição das amostras, alcançando, alguns, até o triplo do valor do que outros. Desta forma, a análise morfológica nos permite fornecer dados úteis na condução do controle da qualidade de drogas vegetais, a fim de proporcionar segurança, à população, quanto ao que estão consumindo.

Palavras chave: "anis verde"; Pimpinella anisum; adulterantes; controle de qualidade; frutas medicinais

\section{INTRODUCCIÓN}

En Paraguay, la utilización de plantas medicinales es una costumbre muy arraigada en la población, en efecto, en la actualidad las generaciones más jóvenes van adquiriendo la costumbre de tomar "tereré", que es un hábito importante para el uso de plantas medicinales, se mantienen así, dos costumbres tan arraigadas y tan nuestras como, el consumo de "tereré" y el uso o empleo de plantas medicinales, que lejos de perderse con el paso de los años, se va arraigando cada vez más como parte de la cultura paraguaya, en todos los niveles sociales (1).

Esta costumbre, es el resultado de una mezcla de la cultura de nuestros antepasados indígenas y la traída por los españoles (2), en efecto, el acervo indígena se agregó al que tenían los europeos sobre sus propias plantas curativas, que fueron progresivamente introducidas en América (3). Actualmente las plantas que se consumen, pueden comercializarse en estado fresco y/o seco, presentadas en forma de droga triturada o polvo grueso, envasadas en bolsitas o en saquitos para preparar decocciones o infusiones (4).

Las plantas medicinales son alternativas relevantes para obtener medicamentos innovadores más seguros y eficaces para diversas patologías humanas (5). Sin embargo, dentro de todo este contexto, surge un importante interrogante: conoce la población lo que está consumiendo? En nuestro país, no existe una política bien definida respecto a las plantas medicinales y fitoterápicos (6), lo 
cual tiene como consecuencia la comercialización de estos productos, sin ningún tipo de control, al no haber una reglamentación que lo establezca o exija. Por otro lado, nuestro país no cuenta con una Farmacopea propia (6), por tanto, debemos regirnos por los estándares establecidos por las Farmacopeas de otros países, por ésta razón es importante que contemos con una Farmacopea propia y que así se establezcan sus propios estándares de calidad en cuanto a este tipo de productos. Para el logro de éste objetivo se requiere, por un lado, el compromiso de las instituciones responsables de realizar esta tarea: el Estado, a través del Ministerio de Salud, la Universidad y el sector privado; y por otro lado, se requiere el concurso de varias disciplinas que trabajen de forma conjunta para realizar trabajos que permitan establecer los caracteres diagnósticos que se deben tener en cuenta a la hora de identificar una droga vegetal.

En Paraguay existe una gran cantidad de especies vegetales empleadas con fines medicinales, según diferentes autores; por ejemplo, el informe del JICA (7), reporta 280 especies medicinales, Basualdo y cols (2003, 2004) (2,8), reportan 266 especies; Pin y cols (2009) (9), reportan más de 300 especies; por citar algunos. Pero, a pesar de gran la cantidad de especies reportadas como medicinales, en nuestro país existen apenas pocos trabajos realizados en el área de la morfología y anatomía, tendientes a aportar herramientas para la identificación de estas plantas medicinales.

Entre las especies empleadas con fines medicinales, en nuestro país, se encuentra el "anís verde", que se utiliza como digestivo, (2-10), antiflatulento, $(2,8)$, expectorante, carminativo, tónico estomacal, para aumentar la secreción láctea de la mujer, como regulador menstrual (9). En el aceite esencial se ha identificado como principal componente el anetol, además, polifenoles, flavonoides y otros compuestos (11).

Se necesitan trabajos que demuestren que estos productos pueden ser pasibles de sufrir adulteración, o bien que poseen materias extrañas no permitidas o que están fuera del rango establecido; y que se aporten herramientas que permitan identificar estos adulterantes. A fin de contribuir con el aporte de tales herramientas, en el control de calidad de drogas vegetales; el presente trabajo tuvo por objetivo identificar y caracterizar morfológicamente, las especies adulterantes en los frutos de "anís verde", Pimpinella anisum, comercializados en las principales herboristerías de Asunción y Gran Asunción.

\section{MATERIALES Y MÉTODOS}

Adquisición de las muestras. Se adquirieron las muestras de frutos de "anís verde", de las principales herboristerías de Asunción y Gran Asunción. Se han encontrado en total 8 marcas que se comercializan como frutos de "anís verde", de cada marca comercializada se compró tres muestras de un mismo lote.

Cada marca fue codificada con una letra del alfabeto: Muestra A, Muestra B, etc. Se compraron las muestras en tres épocas diferentes, siempre de las mismas herboristerías. La primera toma fue en el mes de abril, la segunda en el mes de julio y la tercera en el mes de octubre del año 2015.

Análisis de las muestras. Una muestra de cada lote se conservó como contra muestra y las otras dos fueron sometidas al análisis, previo muestreo por el método del cuarteo (Figura 1). Cada muestra fue observada con el microscopio estereoscópico para identificar, por un lado, los frutos de "anís verde", y por otro lado, las materias extrañas -considerando como materia extraña, la definición de la octava edición de la Farmacopea Argentina- (12); y otras especies presentes, consideradas como adulterantes; éstas fueron caracterizadas e identificadas taxonómicamente, según la base de datos del Missouri Botanical Garden y del Instituto Darwinion $(13,14)$. Se procedió, además, al registro fotográfico de los adulterantes que se observaron en los frutos medicinales objeto de este estudio.

Por otro lado; como anexo a este control, se estableció una relación entre el costo en guaraníes por gramo de muestra y la calidad de la misma, en relación a la presencia o ausencia de adulterantes.

\section{RESULTADOS Y DISCUSIÓN}

Estos productos se presentan comúnmente en el mercado, en bolsitas de polietileno, en diferentes presentaciones, que van de entre $15 \mathrm{~g}$ y $50 \mathrm{~g}$, algunos se presentan en bolsitas de $100 \mathrm{~g}$. Solo una de las muestras se presenta en un envase de plástico con tapa dosificadora.

En todas las muestras adquiridas, se ha observado que los caracteres morfológicos reportados por la Farmacopea Argentina (12), para el "anís verde", coinciden con los caracteres morfológicos observados en la especie presente en las muestras analizadas, por tanto se puede afirmar que se trata de Pimpinella anisum, de la familia Apiaceae, que es la especie reconocida como "anís verde", por dicha Farmacopea (Figuras 2A-C). 


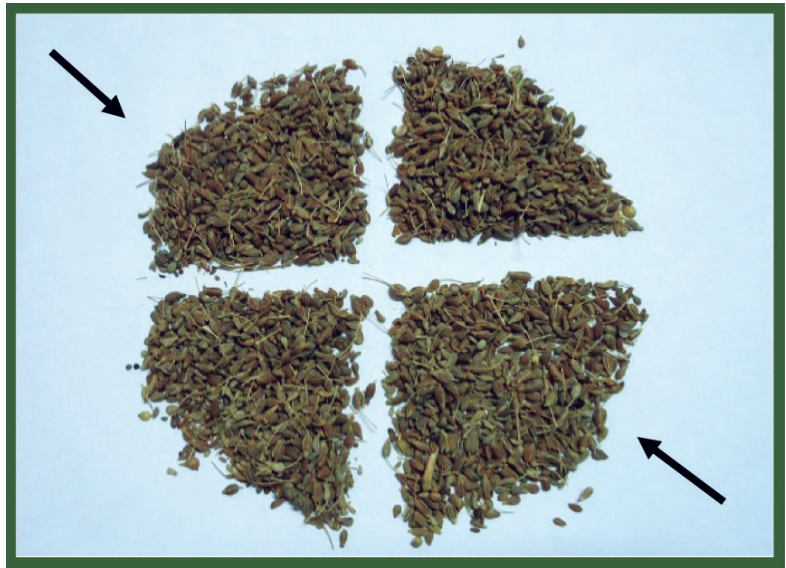

Figura 1. Frutos de "anís verde", Pimpinella anisum. Muestreo por el método del cuarteo. Las flechas indican las porciones de las cuales se toman las muestras.

Sin embargo, en las muestras, se han identificado, además, la presencia de materias extrañas, y semillas y frutos de otras especies, es decir, adulterantes.

En la Tabla 1 se indican las muestras adquiridas, la descripción de la materia extraña (Figura 3) y los adulterantes encontrados. La presencia de materias extrañas en drogas vegetales, es muy común, y de hecho, las Farmacopeas establecen un límite para ellas, ya que es muy difícil que la droga vegetal pueda estar exenta de este tipo de materias. Según la Farmacopea Argentina (12), se considera materia extraña a cualquier parte de la droga vegetal que no esté comprendida en la definición o en la descripción de la monografía correspondiente; las drogas deben estar libres de hongos, de insectos y de otras contaminaciones de origen animal; y salvo que se indique lo contrario, el porcentaje de elementos extraños no debe ser superior al $2 \%$. Por ejemplo, en el caso del fruto del

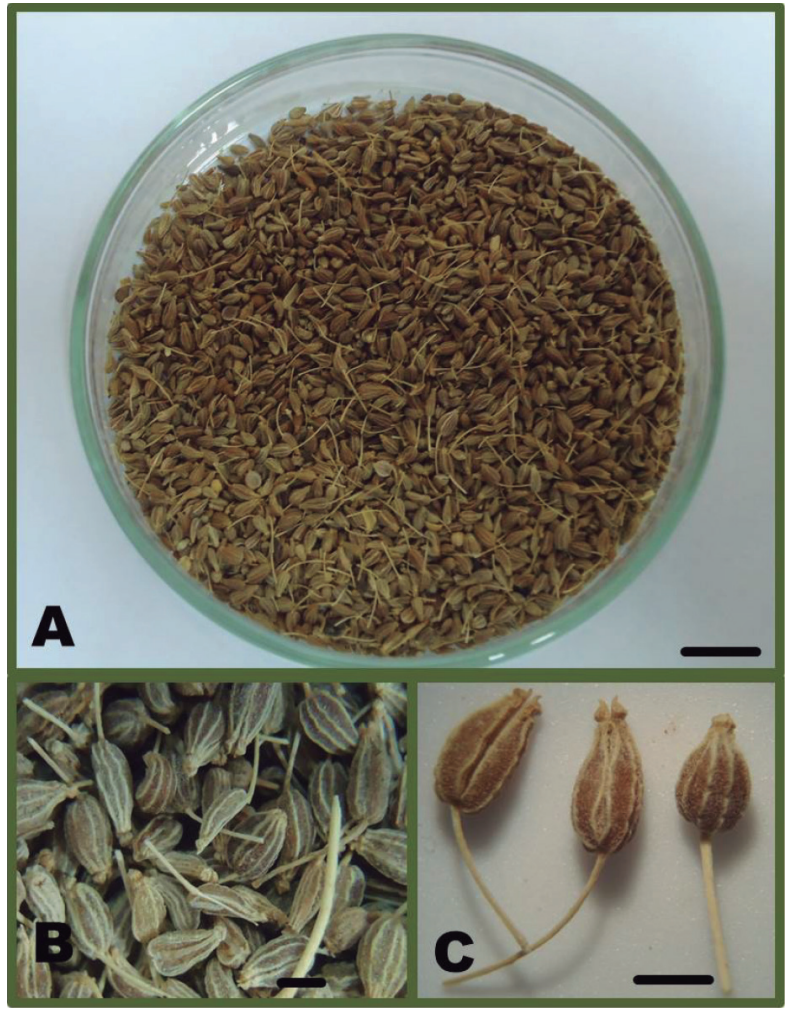

Figura 2. Pimpinella anisum. A. Muestra en caja de Petri. B. Vista con microscopio estereoscópico. C. Vista ampliada de los frutos. Escalas: $A=1 \mathrm{~cm}, B$ y $C=1 \mathrm{~mm}$.

"anís verde", las materias extrañas pueden ser restos de tallos, hojas, o flores de la misma planta, y pueden estar presentes hasta en un 3\%. Es importante aclarar que no toda materia extraña representa en si una adulteración, por ello se establecen límites para ella, de lo contrario estas no deberían estar presentes en las muestras.

Tabla 1. Identificación de materia extraña encontrada en las muestras analizadas de fruto de "anís verde", Pimpinella anisum.

\begin{tabular}{|c|c|c|}
\hline Fruto: ANíS & Descripción de materia extraña encontrada & Adulterante \\
\hline Muestra A & - Pedúnculos & - Restos de tallitos \\
\hline Muestra B & - Restos de tallitos & - Semilla de sésamo \\
\hline Muestra C & - Restos de tallitos & - Fruto de "kuratú" \\
\hline Muestra D & - Pedúnculos & - Semilla de "sésamo" \\
\hline Muestra E & - Restos de tallitos & - Semilla de sésamo \\
\hline Muestra F & - Restos de tallitos & - Fruto de "kuratú" \\
\hline Muestra G & - Restos de tallitos & - Fruto de "kuratú" \\
\hline Muestra H & - Restos de tallitos & - Fruto de "kuratú" \\
\hline
\end{tabular}




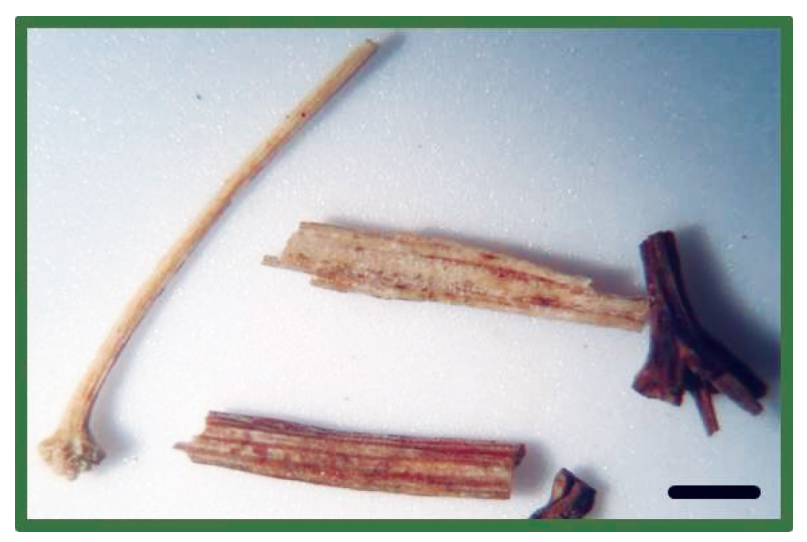

Figura 3. Algunas de las materias extrañas observadas en las muestras de "anís verde" analizadas: pedúnculos y restos de tallo. Escala=1 mm.

Debido a que no se han observado diferencias sustanciales en el porcentaje de materia extraña en las diferentes muestras adquiridas en las tres épocas de colecta, en la Figura 4, se reporta el valor promedio de cada muestra, de las tres colectas.

El promedio de materia extraña observado es de 1,95\%. El valor máximo observado fue de 3,46\% para la muestra A, seguido de la muestra $\mathrm{H}$, con $3,18 \%$, valores que se encuentran por encima del máximo permitido, que es de 3,0\%, para el "anís verde", según la Farmacopea Argentina. Las demás muestras presentan valores por debajo del límite máximo permitido. Las materias extrañas encontradas están constituidas por restos de tallitos y pedúnculos, según se puede observar en la Tabla 1.

Por otro lado, además, de estas materias extrañas propiamente dichas, se han encontrado semillas y frutos, que no corresponden al "anís verde", que pueden ser considerados como adulterantes de los frutos de la especie en estudio. Estos adulterantes han sido identificados como: semilla de "sésamo", Sesamun indicum y frutos de "kuratú", Coriandrum sativum. La presencia y distribución de estos, en las diferentes muestras, se pude apreciar en la Tabla 1. Es de destacar que, en general, solo una de estas especies se encuentra como adulterante, en las muestras de "anís verde", siendo el fruto de "kuratú", la especie más frecuente, encontrándose en seis de las ocho muestras; mientras que la semilla de "sésamo" se ha encontrado en tres muestras y de éstas, una de ellas, específicamente la muestra B, presentó ambas especies.

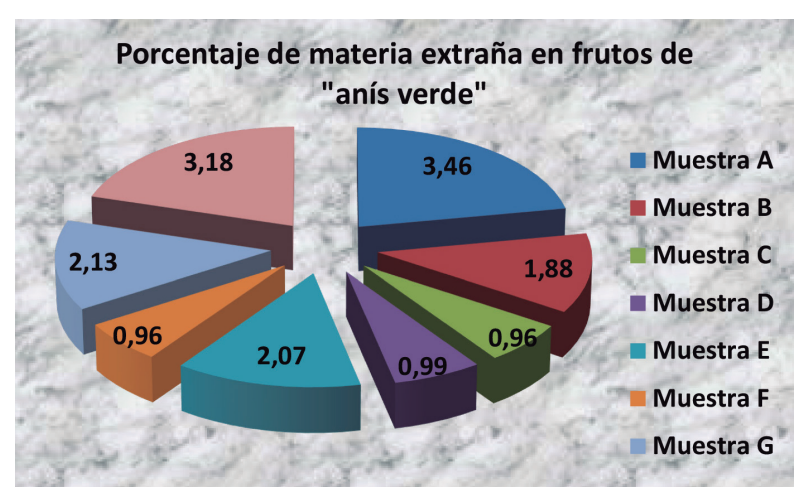

Figura 4. Porcentaje de materia extraña en las muestras de fruto de "anís verde", Pimpinella anisum.

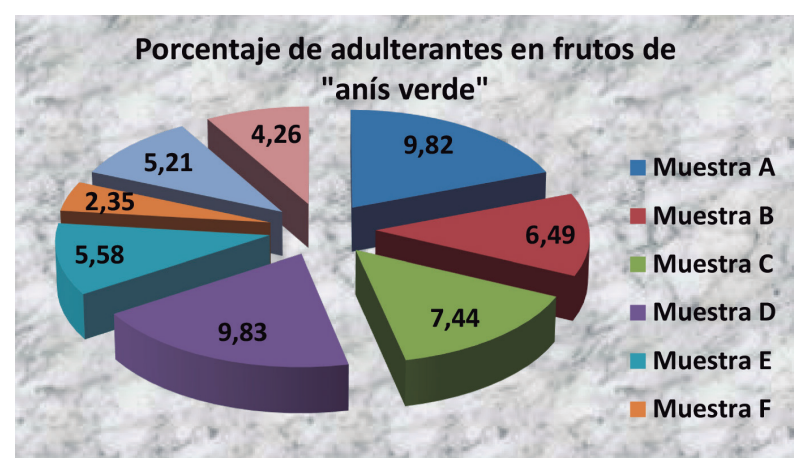

Figura 5. Porcentaje de adulterantes en cada muestra de "anís verde" analizada.

Es de destacar que, en general, solo una de estas especies se encuentra como adulterante, en las muestras de "anís verde", siendo el fruto de "kuratú", la especie más frecuente, encontrándose en seis de las ocho muestras; mientras que la semilla de "sésamo" se ha encontrado en tres muestras y de éstas, una de ellas, específicamente la muestra B, presentó ambas especies. Los porcentajes de estos adulterantes en cada una de las muestras analizadas, se ilustra en la Figura 5.

A fin de poder diferenciar los frutos del "anís verde", de las especies que pueden estar presentes como adulterantes, se presenta a continuación una breve caracterización de dichas especies.

\section{Caracterización de las especies adulterantes encontradas \\ Nombre común: "sésamo", (Figura 6A-B) \\ Nombre científico: Sesamun indicum L. \\ Familia: Pedaliaceae \\ Órgano: Semilla}

Descripción: semillas pequeñas con forma de gota, testa opaca, levemente reticulada, de color marrón muy claro, algunas con punteaduras negras, con borde marcado, arilo visible. 
Nombre común: "kuratú", (Figura 7.A-B)

Nombre científico: Coriandrum sativum L.

Familia: Apiaceae

Órgano: Fruto

Descripción: Esquizocarpo completo, de forma redondeada, formado por dos mericarpos; de color marrón claro, costillas primarias marcadas y de un color más claro lo que las hace más evidentes, que las costillas secundarias, ápice bien marcado, olor característico.
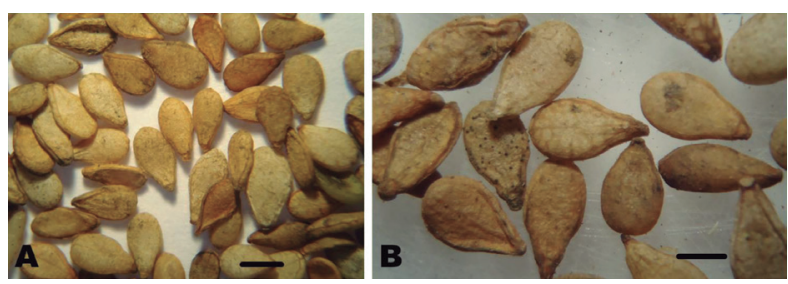

Figura 6. Semillas de "sésamo", Sesamum indicum, adulterante encontrado en las muestras de anís, analizadas. A. Vista con microscopio estereoscópico. B. Vista ampliada. Escalas: A y B=1 mm.
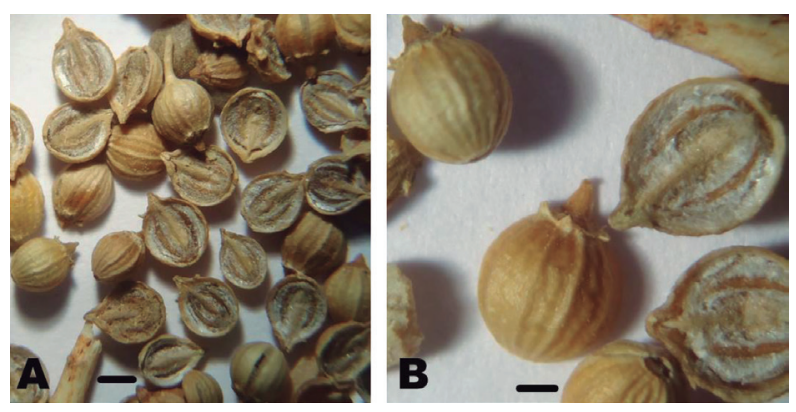

Figura 7. Frutos de "kuratú", Coriandrum sativum, adulterante encontrado en las muestras de anís, analizadas. A. Vista con microscopio estereoscópico. B. Vista ampliada. Escalas: $A=2 \mathrm{~mm}, B=1 \mathrm{~mm}$.

Si bien las especies adulterantes encontradas, no representan en sí, en general, ningún peligro para la salud de las personas, al no estar declaradas en la etiqueta del producto, éstas no deben estar presentes en los mismos. Una muestra que contiene en su composición una especie que no corresponde a lo declarado en etiqueta, debe ser rechazada, y por supuesto no estar a la venta. Por otro lado, existen personas que podrían ser alérgicas a una de estas especies adulterantes, y al no estar declaradas la persona las consume, confiando en lo declarado en etiqueta, pudiendo ocasionarle en ese caso reacciones alérgicas de diversa gravedad.

En la Figura 8 se representan las diferentes muestras y el costo en guaraníes por g de cada una. Esto, a fin de establecer una relación costo/calidad del producto, en relación a los parámetros analizados en este trabajo, específicamente la presencia de adulterantes y otras materias extrañas.

Es interesante notar en la Figura 8, que las muestras analizadas se ofertan en el mercado a diferentes precios; sin embargo, según la Figura 5 todas presentan en su composición especies adulterantes, específicamente, semillas de "sésamo" y frutos de "kuratú" (Tabla 1). Así, se observa que la Muestra $\mathrm{H}$, es la de mayor precio, llegando a costar incluso tres veces más que otras muestras como la Muestra $\mathrm{F}$, que es la más económica, pero que según la Figura 4 es la que presenta menor porcentaje de materia extraña y menor porcentaje de adulterantes (Figura 5). Así que, mal podría pensar el consumidor, que al pagar un mayor precio por una marca determinada de un producto, en este caso, "anís verde", está asegurándose que el mismo es de mejor calidad, que otra marca que tiene menor precio. De manera que mayor precio y mejor calidad, no siempre van de la mano.

Todas las muestras analizadas corresponden a marcas diferentes, sin embargo, todas presentan la misma calidad, en relación a la presencia de adulterantes, incluso, los mismos adulterantes; por tanto sería interesante saber si estas empresas, tiene un proveedor común, o cada una tiene proveedores diferentes. Al respecto se deben hacer dos consideraciones: primero, el "anís verde", no es originario de Paraguay y debe ser importado, por tanto queda la duda de si la adulteración es de origen, o si fue hecha en el país, tal vez, con el fin de abaratar costos; segundo, en nuestro país existen, aunque no en grandes cantidades, cultivos de "anís verde" (15), así que habría que saber sí son estos los frutos de "anís verde" procedentes de estos cultivos los que se comercializan a nivel interno o si solo se exportan.

En Paraguay no existen muchos trabajos orientados a la identificación de adulterantes en productos medicinales. Por ello, es importante que las diversas disciplinas de esta área, se aboquen a la tarea de realizar trabajos de este tipo que puedan brindar herramientas y datos útiles a las autoridades de aplicación, encargadas de velar por la calidad de este tipo de productos, a fin de brindar a la población seguridad en lo que está consumiendo.

Por todo lo mencionado y lo reportado en este trabajo, que de hecho, es apenas una pequeña parte de lo que hace al control de calidad de drogas vegetales y fitoterápicos, es necesaria la realización del control rutinario de este tipo de productos, por parte de la autoridad de aplicación, en este caso el Ministerio de Salud Pública y Bienestar Social, de modo a resguardar, por un lado, el derecho de los consumidores a tener acceso a productos de calidad que satisfagan sus necesidades y por otro lado, la salud misma de estos consumidores, ante la posibilidad de estar expuestos a productos comercializados sin los controles de rigor exigidos, que pueden contener especies adulterantes que podrían ser incluso tóxicas. 


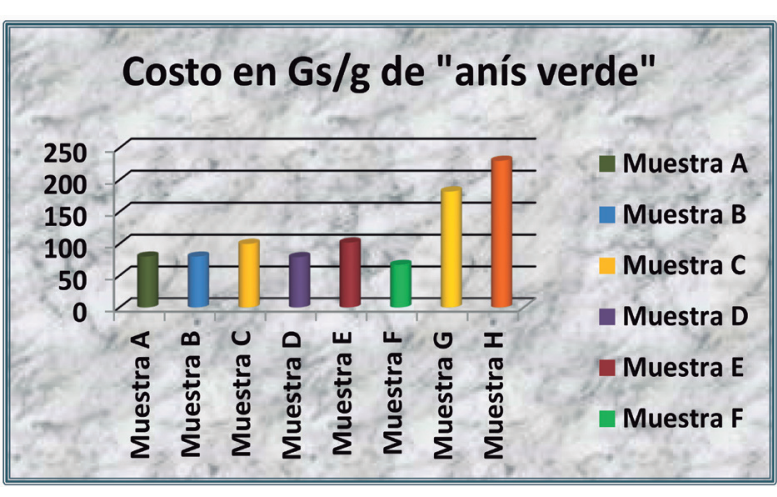

Figura 8. Representación del costo en guaraníes por cada gramo de cada una de las muestras de "anís verde", analizadas.

\section{CONCLUSIÓN}

Las muestras comercializadas como frutos de "anís verde", en las principales herboristerías de Asunción y Gran Asunción, corresponden a la especie Pimpinella anisum (Apiaceae); 2 muestras presentan un porcentaje de materia extraña superior a lo establecido en la Farmacopea Argentina, y todas las muestras presentan especies adulterantes, entre las cuales se han identificado dos especies: semilla de "sésamo", Sesamun indicum y fruto de "kuratú", Coriandrum sativum; las

\section{REFERENCIAS BIBLIOGRAFICAS}

1. Degen, R, González, Y. Plantas utilizadas en la medicina popular paraguaya como antiinflamatorias. Bol Latinoam Caribe Plant Med Aromat 2014; 13(3):213-231.

2. Basualdo, I, Soria, N, Ortiz, M, Degen, R. Uso medicinal de plantas comercializadas en los mercados de Asunción y Gran Asunción, Paraguay. Revista de la Sociedad Cien-tífica del Paraguay. Tercera Época. Año VII. 2003; 14:5-22.

3. Arenas, P. Etnobotánica lengua maskoy. Fundación para la educación, la ciencia y la cultura (FECIC). Buenos Aires, Argentina. 1981.

4. Degen, R, Basualdo, I, Soria, N. Comercialización y conservación de especies vegetales medicinales en Paraguay. Revista Fitoterapia 2004; 4(2):139-148.

5. Ibarrola, DA, Degen de Arrúa, RL. Catálogo ilustrado de 80 Plantas Medicinales del Paraguay. Facultad de Ciencias Químicas, UNA \& Agencia de Cooperación Internacional del Japón (JICA). 2011. Pp. 178.

6. Degen, R, González, Y, Amarilla, A. Legislación sobre plantas medicinales y fitoterápicos en Paraguay: una tarea pendiente. Bol Latinoam Caribe Plant Med Aromat 2008; 7:1-5.

7. JICA. Report on Cooperation in Study (Chemical and Pharmaceutical Study on Herbs) with Paraguay. 1987. Pp. 178. cuales han sido caracterizadas para su diferenciación e identificación. Se observó, además, que todas las muestras presentaron en $\mathrm{su}$ composición algunos de estos adulterantes o incluso ambos, sin embargo, todas se comercializan a diferentes precios, algunas incluso al triple del valor que otras. La determinación de posibles adulteraciones en drogas vegetales, junto con la identificación botánica de las mismas, es uno de los primeros pasos en el control de calidad de plantas medicinales y fitoterápicos. De esta manera, el análisis morfológico, se constituye en una herramienta muy útil que puede aportar los caracteres de diagnóstico que permitan caracterizar una especie vegetal, y sus posibles adulterantes, en el control de calidad de drogas vegetales.

\section{AGRADECIMIENTOS}

Las autoras agradecen a la Dirección General de Investigación, Ciencia y Tecnología, del Rectorado de la Universidad Nacional de Asunción, por la financiación del Proyecto: "Identificación de adulterantes y materias extrañas en frutos medicinales, comercializados en principales herboristerías de Asunción y Gran Asunción, como aporte para el control de calidad de drogas vegetales", dentro de cuyo marco se desarrolló el presente trabajo.
8. Basualdo, I, Soria, N, Ortiz, M, Degen, R. Plantas medicinales comercializadas en los mercados de Asunción y Gran Asunción, Parte I. Rojasiana 2004; 6(1):95-114.

9. Pin, A, González, G, Marín, G, Céspedes, G, Cretton, S, Christen, P, Rouget, D. Plantas Medicinales del Jardín Botánico de Asunción. Municipalidad de Asunción, Asociación Etnobotánica Paraguaya. Asunción, Paraguay. 2009.

10. Soria, N, Basualdo, I. Medicina Herbolaria de la comunidad Kavaju Kangue, Departamento de Caazapá, Paraguay. 2005.

11. Witchl, M, Anton, R. Plantes thérapeutiques. Edition Tec \& Doc. París, Francia. 2003.

12. Farmacopea Argentina. Capítulo: Métodos Generales de Farmacognosia. Octava Edic. Buenos Aires, Argentina. 2008.

13. www.tropicos.org. Base de datos del Missouri Botanical Garden, Estados Unidos. Consultado el 18 de febrero de 2017.

14. www.darwinion.com.ar. Base de datos del Instituto de Botánica Darwinion, Argentina. Consultado el 14 de noviembre de 2017.

15. Fundación Cardajin. Informe sobre la situación de las plantas medicinales y aromáticas en Paraguay. 2008. 